\title{
The Surgical Management of Dysthyroid Related Eyelid Retraction Using Mersilene Mesh
}

\author{
R. N. DOWNES ${ }^{1}$ and K. JORDAN ${ }^{2}$ \\ Cambridge
}

\begin{abstract}
Summary
The use of Mersilene mesh as a spacer material in the surgical management of seven patients with dysthyroid-related eyelid retraction is presented. To date, fourteen eyelids have been operated on using this material with an average follow-up of nine months. It appears that Mersilene mesh is a realistic alternative to preserved sclera in the surgical management of such cases.
\end{abstract}

The use of synthetic mesh materials has not previously been described in the English literature in eyelid surgery. The experiences reported by other disciplines ${ }^{1-6}$ using Mersilene mesh suggest that it may be a valuable substitute for preserved sclera in the management of dysthyroid related lid retraction.

A prospective clinical trial was undertaken using Mersilene mesh as a lid spacer material in cases of upper and lower eyelid retraction arising from dysthyroid eye disease.

\section{Materials and Methods}

Seven patients with dysthyroid eye disease had Mersilene mesh inlay procedures performed (Table I). Surgery was undertaken on a total of fourteen eyelids; four upper and ten lower lids. Previous eye surgery had been performed in five patients (Table II). The patients were fully evaluated pre-operatively with particular emphasis upon superior and inferior scleral show in the primary position of gaze (Table III). All patients complained of ocular discomfort and poor cosmesis. All gave informed consent to the planned surgical procedures.
In upper eyelid retraction a standard anterior approach was performed..$^{7.8}$ The levator-aponeurosis complex was identified and mobilised with careful attention to complete division of the lateral horn. The posterior

Table I Patient details

$$
\begin{gathered}
7 \text { patients }\left\{\begin{array}{l}
5 \text { Female } \\
2 \text { Male }
\end{array}\right. \\
\text { Age range } 25 \mathrm{yr}-52 \mathrm{yr} . \\
\text { Average } 41 \mathrm{yr} .
\end{gathered}
$$

All clinically and biochemically euthyroid for at least 6 months at the time of operation.

Table II Previous ophthalmic surgery

\begin{aligned} & \hline 1 Patient -$($ L) orbital decompression for \\ & compressive optic neuropathy \\ &- Bilateral inferior rectus recessions \\ & 2 Patients - Bilateral lateral tarsorrhaphies \\ &$(3$ out of 4 opened spontaneously post- \\ & operatively $) \\ & 1$ Patient - Bilateral levator myotomies \\ & 1 Patient - Upper lid scleral inlay \end{aligned}

From: 'Ophthalmic Department, New Addenbrookes Hospital, Cambridge and 2Ophthalmic Department, Princess of Wales Hospital, RAF Ely, Cambs.

Correspondence to: Sqn Ldr R N Downes, Consultant Ophthalmic Surgeon, Princess Mary's Hospital, RAF Halton, Aylesbury, Bucks HP22 5PS 
Table III Pre-operative measurements (in primary position of gaze)

Upper lids

Range $\quad 4-5 \mathrm{~mm}$ superior scleral show Average $4.7 \mathrm{~mm}$ superior scleral show

Lower lids

Range $\quad 2-5 \mathrm{~mm}$ inferior scleral show Average $3.5 \mathrm{~mm}$ inferior scleral show

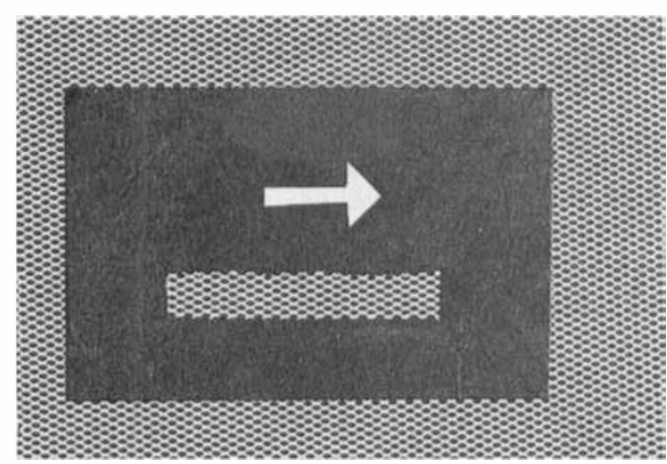

Fig. 1. Close up photograph of Mersilene mesh spacer, demonstrating mesh configuration. The mesh is cut horizontally in the direction arrowed.

plane of dissection was between the levator complex and Muller's muscle such that the latter structure with conjunctiva remained attached to the superior tarsal border. A spacer of Mersilene mesh was fashioned based upon the measurements widely adopted for sclera $^{8.9}$ i.e. horizontal dimensions equal to those of the tarsus and vertical dimensions twice the amount of pre-operative scleral show laterally, one and one half times the scleral show centrally and medially. Mersilene mesh has a specific fibre-junction configuration. To avoid excessive horizontal laxity the mesh is cut as illustrated in Figure 1. The mesh was sutured to the free inferior border of the levator complex superiorly and the superior tarsal border inferiorly using continuous $6 / 0$ vicryl. Interrupted absorbable sutures were used to close the skin and reform the skin crease. A central upper lid traction suture was taped to the cheek for 48 hours postoperatively.

In lower eyelid retraction a conventional posterior approach was adopted. ${ }^{7.8}$ The conjunctiva was incised along the lower tarsal border and extensively undermined down to the fornix. The lower lid retractors were divided from the tarsal border exposing the suborbicularis plane, and mobilised using blunt dissection inferiorly in this plane. A Mersilene mesh spacer was cut to size using dimensions adopted for sclera, ${ }^{8.9}$ with horizontal dimensions equal to those of the tarsus and a vertical height of twice the pre-operative scleral show. The mesh was sutured inferiorly to the free border of the lower eyelid retractors and superiorly to the inferior tarsal border using $6 / 0$ vicryl. The conjunctiva was sutured to the tarsal margin so that the mesh was completely covered, using a continuous vicryl suture. Full thickness bolster sutures were used in some cases. Two lower lid traction sutures, one medial and one lateral, were taped to the brow for 48-72 hours post-operatively. Lateral and/or medial tarsorrhaphies were performed in certain cases.

All surgery was performed under general anaesthesia.

\section{Results}

A significant improvement in eyelid position was achieved in all cases (Table IV). Figures 2-5 illustrate two patients. To date, the eyelid height has been maintained in all patients with an average follow-up of nine months (range 5-16 months). All the patients were pleased with the post-operative cosmetic result and the majority reported a marked improvement in, or abolition of, ocular discomfort. Signifi-

Table IV Post-operative measurements (in primary position of gaze)

Upper lids

(Total 4)

All within $1 \mathrm{~mm}$ of the superior limbus

1 -significant lateral peaking

Lower lids

(Total 10)

Range $\quad 0-2 \mathrm{~mm}$ inferior scleral show

Average $0.6 \mathrm{~mm}$ inferior scleral show

Table V Complications 


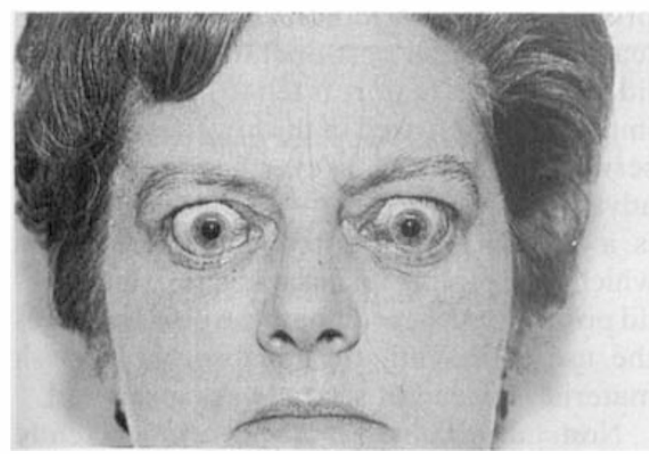

Fig. 2. Pre-operative appearance.

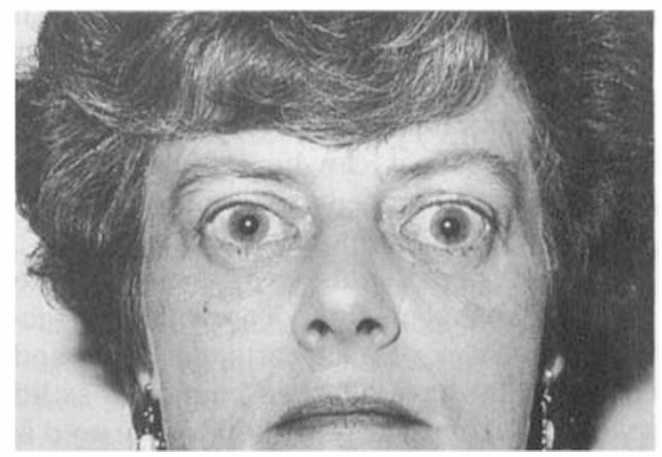

Fig. 4. Pre-operative appearance.

cant lateral peaking of the upper eyelid occurred in one patient who had a similar appearance of the contralateral lid following a scleral inlay. Subsequent bilateral upper lid explorations were performed with adequate release of the lateral horns: satisfactory upper eyelid contours resulted.

A number of complications were encountered (Table $\mathrm{V}$ ) the most troublesome of which was a chronic, unilateral papillary conjunctivitis in one patient who had undergone bilateral lower lid Mersilene inlays. Topical antibiotics and steroids were required with gradual resolution of the condition over several months. Three patients noticed localised tenderness of the lids. No cause was found in two cases which resolved spontaneously. The third case demonstrated a small area of mesh exposure through the conjunctiva of the upper lid. One patient had a

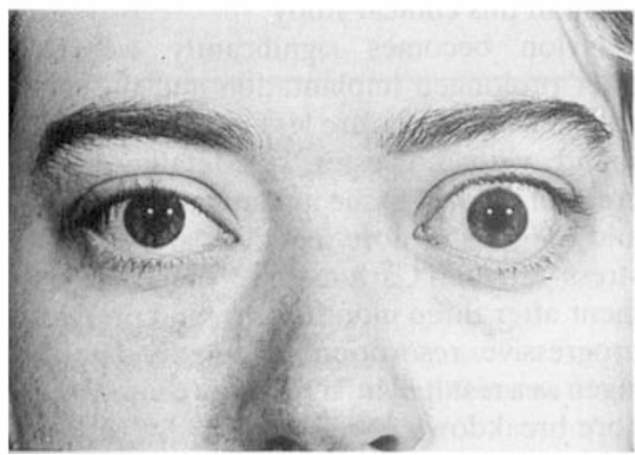

Fig. 3. Post-operative appearance of patient illustrated in Figure 2 four months after insertion of Mersilene mesh spacers in all four eyelids.

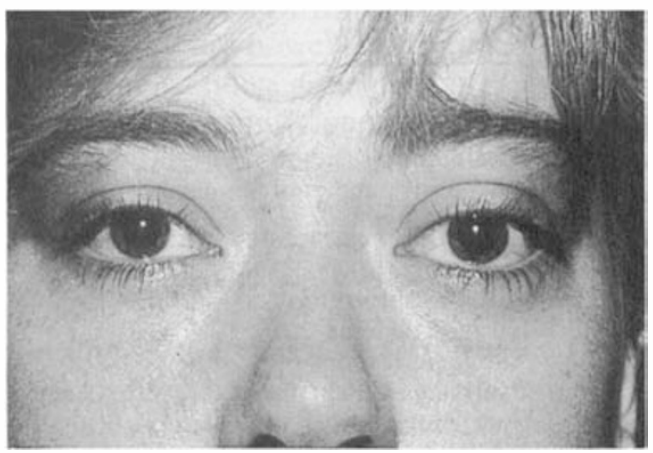

Fig. 5. Post-operative appearance of patient illus trated in Figure 4 six months after bilateral lower lid Mersilene mesh inlay procedures and left levator complex recession.

similar although asymptomatic area of lower eyelid mesh exposure. The exposed mesh was excised and covered with conjunctiva in each case with no further problems and no effect on the eyelid position.

\section{Discussion}

The current management of significant lid retraction in dysthyroid eye disease is somewhat controversial. ${ }^{7-15}$ Whilst it is generally agreed that a lid spacer material is required for the correction of $2 \mathrm{~mm}$ or more of lower eyelid retraction no such consensus exists regarding the management of upper eyelid retraction. Some authors believe that an upper eyelid can be satisfactorily lowered, whatever the amount of retraction, with surgery directed towards recessing and/or weakening the levator complex. ${ }^{11-13}$ Others believe that a lid spacer is required to correct in excess 
of $3 \mathrm{~mm}$ of upper eyelid retraction. ${ }^{7.8 .9 \cdot 10.14 .16}$ If a lid can be lowered adequately without resort to the use of foreign materials then so much the better but it is our experience that a more reliable lid height can be achieved with a single operative procedure if a spacer material is used to lengthen the retractor complex.

A variety of materials have been used in the surgical management of eyelid retraction..$^{89,11.16}$ Sclera, fascia, cartilage, tarsus and collagen film have all been employed as lid spacers. The most popular and widely used is preserved sclera. It is easily implanted, inexpensive, usually readily available and has a

\section{Table VI Preserved sclera}

Advantages

Widely used; proven 'track record' Availability

Inexpensive and easily implanted

'Lid stiffener'

Disadvantages

Variable absorption

Lid bulk/oedema

Antigenic response

Overlying skin changes

Cyst formation

Availability; 24-hour preparation

H.I.V. status

Corneal irritation

Table VII Clinical applications of Mersilene mesh

\begin{tabular}{l} 
1. General surgery \\
$\begin{array}{l}\text { - diaphragmatic defects } \\
\text { - hernia repairs }\end{array}$ \\
$\begin{aligned} \text { 2. Vascular surgery } & - \text { vascular prostheses } \\
\text { 3. Orthopaedic surgery } & - \text { ligament/tendon } \\
& - \text { repairs/replacement }\end{aligned}$ \\
\hline
\end{tabular}

Table VIII Mersilene mesh

Advantages
Readily available and inexpensive
Easily prepared, shaped and implanted
Strong, durable and flexible
Provision of permanent scaffolding for fibrovascular
ingrowth
Well tried and tested in various clinical settings
Disadvantages
Foreign material
? Flexible
Probably requires conjunctival cover

proven record. Additionally sclera acts, at least in the initial post-operative period, as a lid spacer. This feature is felt to be particularly important when used in the lower eyelid. Preserved sclera does however have certain disadvantages ${ }^{8.9 .11 .16}$ (Table VI) not least of which is a variable and unpredictable absorption which can result in an unsatisfactory final eyelid position. As a result of these disadvantages the use of a synthetic nonabsorbable mesh material, instead of sclera, was considered.

Non absorbable meshes are currently manufactured using a variety of materials. Polyester mesh appears to have certain advantages which prompted the use of Mersilene mesh in this clinical study.

Nylon becomes significantly weakened after prolonged implantation and the meshcollagen adhesions are less regular than these found with polyester. ${ }^{1.17}$ Metallic meshes create a marked tissue inflammatory response and the sites of fibre junctions are subject to stress fatigue. Carbon fibre materials fragment after three months or so and produce a progressive resorption of surrounding collagen as a result of inflammation caused by the fibre breakdown. ${ }^{3}$

Mersilene mesh is an interlocking polyester fibre mesh. ${ }^{16,17}$ It is manufactured using a machine knitting process which interlocks each fibre, thus preventing unravelling. This allows cutting of the mesh into different shapes and sizes without significantly disrupting adjacent junction sites. Histological findings in animal studies have shown that the mesh is initially covered with a thin fibrous layer. Subsequent fibrovascular ingrowth into the open meshwork occurs, intimately integrating the mesh and contact tissue. Clinical studies have confirmed these findings. ${ }^{1,3.16,17}$ Mersilene mesh has been available for thirty years or so and extensively used in a variety of clinical settings (Table VII). Interestingly, Peyman et al. ${ }^{18.19}$ when studying intraocular lens fixation in rabbit eyes reported fibroblastic and pigmented epithelial cell ingrowth into polyester mesh within five days of implantation resulting in consistent and localised adhesion to the posterior iris. In comparison with teflon, nylon, silk and catgut, polyester mesh produced maximal adhesion with minimal post-operative inflammation. The advan- 
Table IX

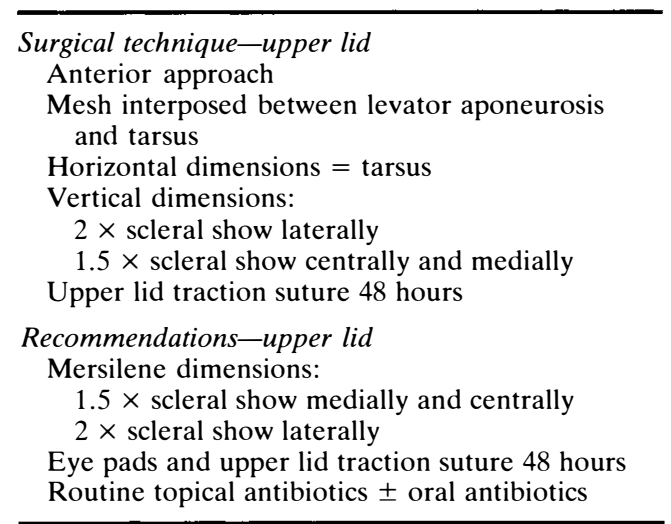

Table X

tages and disadvantages of Mersilene mesh are summarised in Table VIII.

The results using Mersilene mesh compare favourably with those generally reported when sclera has been used as a lid spacer. Although the polyester mesh is relatively thin and flexible this did not seem to be disadvantageous when used in the lower eyelid. Our findings suggest that a relatively rigid, inflexible material such as sclera or cartilage may not be as advantageous as is usually assumed. It has been suggested that permanent medial and lateral canthal support, in the form of lateral tarsorrhaphy and medial canthoplasty, is necessary to obtain a satisfactory lower eyelid position. Some of our

patients expressed concern about resultant cosmesis, particularly of the medial canthal region, when the proposed surgery was outlined. Therefore where possible these procedures were omitted using instead temporary medial and lateral traction sutures, taped to the forehead for 72 hours. The results suggest that in certain cases, usually those with isolated lower eyelid retraction, permanent lateral and medial lid apposition may not be necessary.

Only one patient required re-operation for an unsatisfactory lid position. This was the result of failure to free adequately the lateral horn of the levator complex rather than any inherent problem arising from the use of Mersilene. We did however encounter a number of complications. One patient developed a unilateral chronic papillary conjunctivitis, which did however fully resolve with prolonged topical antibiotic and steroid therapy. The patient concerned had bilateral Mersilene inlays; the contralateral lid gave no such problems. This suggested a local mesh abnormality as the causative factor rather than a generalised reaction to the mesh. When Mersilene mesh is cut with scissors, the divided fibres adjacent to mesh junctions become frayed. It is possible that one or several cut fibre edges traumatised or perforated the overlying conjunctiva (although the latter was not apparent on slit lamp examination) setting up a local conjunctival reaction. Frank exposure of an area of mesh was seen in two cases. In one patient an area of localised mesh exposure through the conjunctiva was apparent on eversion of the upper eyelid. Microscopic examination revealed that the exposed mesh formed part of the lower cut margin of the inlay. It seemed likely that this free margin had eroded through the adjacent conjunctiva. The mesh was carefully trimmed and resutured, with closure of the overlying conjunctiva; the patient has not experienced further problems. The case of asymptomatic localised mesh exposure was complicated by inadvertent removal of the continuous conjunctival suture two weeks post-operatively, although it is possible that limited fibre erosion through the conjunctiva also occurred. Localised mesh excision and resuturing resolved the problem. 
Careful preparation of the Mersilene inlay combined with scrupulous suturing should minimise the likelihood of mesh exposure, but the problem of localised fibre disruption associated with freehand cutting of the mesh still exists. The manufacturers of Mersilene mesh have been approached; they are currently studying alternative means of cutting the mesh and sealing the disrupted fibres in an attempt to obviate this problem.

Two patients were aware of localised eyelid tenderness for several weeks post-operatively although this had no obvious cause and settled spontaneously. Three patients complained of a prolonged and intermittent mucoid discharge. This settled with time and topical antibiotic therapy and was probably related to the slowly absorbable conjunctival suture.

Our recommendations for the use of Mersilene mesh as a lid spacer are outlined in Tables IX and X.

\section{Conclusion}

The results obtained, at least in the short term, are most promising although further clinical evaluation is necessary. If a spacer material in lid surgery is deemed appropriate, this study suggests that Mersilene mesh may be a suitable alternative to preserved sclera.

We would like to thank Mr P G Watson, Mr J Keast Butler and Mr A T Moore for their help and co-operation with this study, and Miss G Mason for her valuable secretarial assistance.

\section{References}

${ }^{1}$ Adler RH and Furne CN: Use of pliable synthetic mesh in the repair of hernias and tissue defects. Surg Gynecol Obstet 1959; 108: 199-206.

${ }^{2}$ Nichols DA: The Mersilene Mesh gauze hammock for severe urinary stress incontinence. Obstet Gynecol 1973, 41: 88-93.

${ }^{3}$ Amis AA: Filamentous implant reconstruction of tendon defects: a comparison between carbon and polyester fibres. J Bone Joint Surg (Br) 1982, 643: 682 .

${ }^{4}$ Bayer I, Feller N, Chaimoff $\mathrm{CH}$ : A new approach to the nipple in Koch's reservoir ileostomy using Mersilene Mesh. Div Colon Rectum (USA) 1981, 24: 428-31.

${ }^{5}$ Adloff $\mathbf{M}$ and Arnaud JP: Surgical management of large incisional hernias by an intra peritoneal Mersilene Mesh and an aponeurotic graft. Surg Gynecol Obstet 1987, 165: 204-6.

${ }^{6}$ Meadows TH and Davies DR: Late reconstruction of the patellar ligament using a dacron ligament implant. J Roy Coll Surg Edin 1987, 32: 322-3.

7 Collin JRO: Ptosis/Corneal Protection. In Collin JRO ed. A manual of systematic eyelid surgery. Edinburgh: Churchill Livingstone 1983, 69-71 and 117-9.

${ }^{8}$ Dryden RM and Doxanas MT: Eyelid malpositions, part I. In McCord Jr. CD ed: Oculoplastic Surgery. New York: Raven Press 1982, 97-105.

${ }^{9}$ Doxanas MT and Dryden RM: The use of sclera in the treatment of dysthyroid eyelid retraction. Ophthalmology 1981, 88: 887-94.

${ }^{10}$ Beyer CK and Albert DM: The use and fate of fascia lata and sclera in ophthalmic plastic and reconstructive surgery. Ophthalmology 1981, 88: 86986.

"Harvey JT and Anderson RL: The aponeurotic approach to eyelid retraction. Ophthalmology 1981, 88: 513-24.

12 Grove AS: Upper eyelid retraction and Graves' disease. Ophthalmology 1981, 88: 499-506.

${ }^{13}$ Putterman AM: Surgical treatment of thyroidrelated upper eyelid retraction. Ophthalmology 1981, 88: 507-12.

${ }^{14}$ Shorr $\mathbf{N}$ and Seiff SR: The four' stages of surgical rehabilitation of the patient with dysthyroid ophthalmopathy. Ophthalmology 1986, 93: 47683.

15 Thaller VT, Kaden K, Lane CM, Collin JRO: Thyroid lid surgery. Eye 1987, 1: 609-14.

${ }^{16}$ Lisman RD and Smith BC: Eyelid surgery for thyrọid ophthalmology. In Smith BC, Della Rocca RC, Nesi FA and Lisman RD eds. Ophthalmic Plastic and Reconstructive Surgery St Louis: CV Mosby Company 1987, Vol 2: 1401-14.

${ }^{17}$ Product sheet-Mersilene Mesh: revised 9/85 from Ethicon Ltd.

18 Peyman GA and Koziol J: Intraocular lens fixation with Dacron mesh: part I. Ophthalmic Surg 1977, 8: $58-64$.

${ }^{19}$ Peyman GA, Koziol J, Janevicius R: Intraocular lens fixation with Dacron mesh: Part II. Ophthalmic Surg 1977, 8: 87-93. 